\title{
Electron Microscopy of In Situ Presolar Silicon Carbide
}

\author{
Rhonda M. Stroud*, Megan O’Grady ${ }^{* *}$, Larry R. Nittler ${ }^{* * *}$, and Conel M. O’D. Alexander ${ }^{* * *}$
}

"Naval Research Laboratory, Code 6371, 4555 Overlook Avenue SW, Washington, DC 20375

** Dept. of Physics and Astronomy, Vanderbilt University, VU Station B 351807, Nashville, TN 37235.

**** Dept. of Terrestrial Magnetism, Carnegie Institution of Washington, 5241 Broad Branch Road NW, Washington, DC 20015

Data gathered from structural, chemical and isotopic studies of presolar grains help astronomers constrain models of stellar evolution. The isotopic properties of presolar $\mathrm{SiC}$ grains are very well studied and indicate that most of the grains formed in the outflows of carbon-rich Asymptotic Giant Branch (AGB) stars [1,2]. Microstructural studies of presolar $\mathrm{SiC}$ are more sparse, but such studies can provide important information about the nebular environment in which the presolar grains formed [e.g., 3]. Initial studies by transmission electron microscopy (TEM) and Raman spectroscopy indicated that most, if not all, isotopically anomalous $\mathrm{SiC}$ grains have the face-centered abic $\beta-\mathrm{SiC}$ structure [4-6]. More recently, Daulton et al. [7] have shown that a significant fraction of sub-micron presolar $\mathrm{SiC}$ grains are of the hexagonal $2 \mathrm{H}$ polytype $(\alpha-$ $\mathrm{SiC}$ ). All of the preceding studies were performed on $\mathrm{SiC}$ grains isolated from their host meteorites by harsh chemical treatments. These treatments have been shown to significantly alter the surface morphology of the grains [8], and might break apart cracked or fissured grains and preferentially destroy less resistant minerals or organic phases associated with the SiC. Here we report the first TEM microstructural study of a presolar $\mathrm{SiC}$ grain identified in situ in a polished meteorite section by $\mathrm{x}$-ray mapping, and extracted for TEM analysis using the focused ion beam lift-out technique [9].

We implemented an x-ray mapping technique, similar to that developed by [10], on a JEOL JXA-8900 electron microprobe. This method exploits the higher $\mathrm{Si} \mathrm{K}-\alpha$ x-ray yield from $\mathrm{SiC}$ compared with other $\mathrm{Si}$ bearing phases in chondrite matrices. In contrast to [10], we used a multi-detector wavelength-dispersive xray system, resulting in a higher signal-to-noise ratio. Elemental maps of $\mathrm{C}, \mathrm{Mg}, \mathrm{Si}, \mathrm{S}$ and $\mathrm{Ca}$ (or $\mathrm{Al}$ ) were obtained and potential $\mathrm{SiC}$ grain candidates identified by high counts of $\mathrm{Si}$ and $\mathrm{C}$ and low counts of the other elements. Accelerating voltage, beam current and integration times were chosen to optimize detection efficiency of micron-sized $\mathrm{SiC}$ grains while minimizing the number of false candidates. We obtained nine $500 \times 500 \mu \mathrm{m}$ maps $\left(2.25 \mathrm{~mm}^{2}\right)$ from a polished section of the Cold Bokkeveld meteorite. One SiC grain, of size $\sim 2.5 \times 2 \mu \mathrm{m}$, was identified.

Diffraction studies of the $\mathrm{SiC}$ grain show it to be the cubic $\beta$-SiC phase, consistent with prior reports for the majority of presolar $\mathrm{SiC}$ grains $>1 \mu \mathrm{m}$ [4-6]. In contrast, most commercially available synthetic $\mathrm{SiC}$ powders have hexagonal structures. The selected-area diffraction pattern (SADP) from the [110] zone (Fig. 1a) shows heavy streaking along <111> directions, indicative of a high density of stacking faults, also consistent with prior observations. Extra spots are present that may indicate twinning. In addition, the SADP shows two rings, at $0.342 \mathrm{~nm}$ and $0.212 \mathrm{~nm}$, corresponding to the (002) and (100) d-spacings of graphite, respectively. Dark field imaging ( $\mathrm{g}=(111)$ ) of the $\mathrm{SiC}$ grain (Fig. 1b) highlights the high density of stacking faults, and also reveals the presence of sub-grains. Some of these sub-grains are believed to be the graphite indicated by the rings in the SAD pattern. Other sub-grains, which appear elongated in the (001) direction 
of the primary $\mathrm{SiC}$ are likely $\mathrm{SiC}$ twins. The possibility of other carbides, e.g., $\mathrm{TiC}$, is refuted by energy dispersive X-ray measurements, which showed only Si and C.

The SiC grain boundaries are sharply faceted along $<111\rangle$ directions. There is no evidence for an oxide layer at the grain boundaries, although the possibility of a very thin oxide layer $(<1 \mathrm{~nm})$ cannot be ruled out. The lack of a thick oxide layer indicates that the grain was not exposed to hot $(>1000 \mathrm{~K})$ nebular gas for any significant length of time [11]. The surrounding matrix consists primarily of sub-micron phyllosilicate grains, with no apparent crystallographic relationship to the SiC. This confirms the lower-resolution SEM observation of [10] that presolar $\mathrm{SiC}$ is sited as isolated grains in the matrix without other associated mineral phases.

The TEM results indicate that the $\mathrm{SiC}$ formed by rapid vapor-phase condensation, trapping pre-existing graphite grains in random orientations. This crystallization sequence, i.e., graphite followed by other carbides, is expected for the $\mathrm{C} / \mathrm{O}$ ratios and pressures found in the outer atmospheres of AGB stars [3]. However, to our knowledge, this is the first observation of graphite enclosed in presolar $\mathrm{SiC}$ [12].

\section{References}

[1] P. Hoppe and U. Ott in Astrophysical Implications of the Laboratory Study of Presolar Materials, AIP Conf. Proc. 42 (1997) 27.

[2] E. Zinner Ann. Rev. Earth Planet. Sci. 26 (1998) 147.

[3] T.J. Bernatowicz et al. Astrophysical J. 47 (1996) 760.

[4] T.J. Bernatowicz et al. Nature 330 (1987) 728.

[5] A. Virag et al. Geochem. Cosmochem. Acta 56 (1992) 1715.

[6] T.J. Bernatowicz et al. LPSC XXIII (1992) 91.

[7] T. Daulton et al. LPSC XXXIII (2002) in press.

[8] T.J. Bernatowicz et al. LPSC XXXI, Abstract \#1238 (CD-ROM) (2000).

[9] P.J. Heaney et al. Amer. Mineral. 86 (2001) 1094.

[10] C.M.O'D. Alexander et al. Nature 348 (1990) 715.

[11] R.A. Mendybaev et al. LPSC XXX, Abstract \#1990 (CD-ROM) (1999).

[12] We thank Bill Minarik and the CIW summer intern program for the support of Megan O'Grady. This work was partially supported by ONR, and NASA grant NAG5-4328.
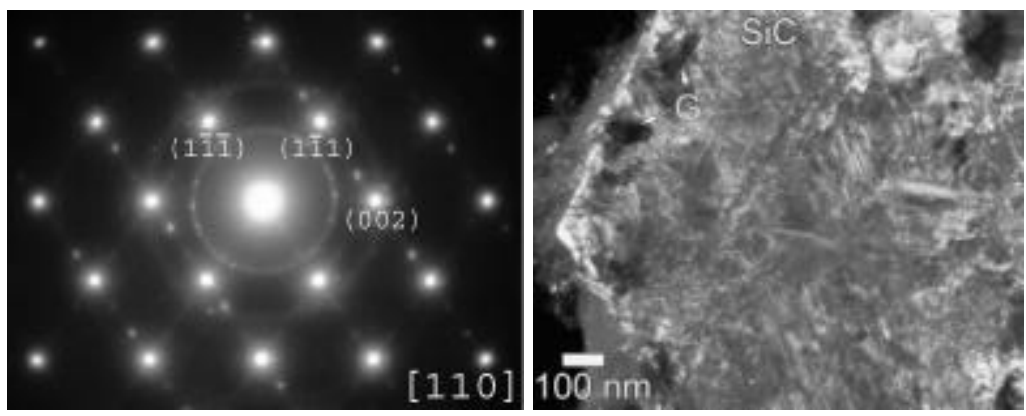

FIG. 1. (a) Selected area diffraction of a presolar SiC grain. (b) Dark field image of a presolar SiC grain. Possible graphite sub-grains are indicated by " $G$ ". 